\title{
Lung Function Normalisation with Indacaterol Acetate/ Glycopyrronium Bromide/Mometasone Furoate in Patients with Asthma
}

\author{
Kenneth R. Chapman ${ }^{1,2} \cdot$ Henrik Watz $^{3} \cdot$ Dave Singh $^{4} \cdot$ Jens M. Hohlfeld ${ }^{5}$ Zuzana Diamant ${ }^{6,7,8} \cdot$ leuan Jones $^{9}$. \\ Hanns-Christian Tillmann ${ }^{10} \cdot$ Ivan Nikolaev ${ }^{9}(0)$
}

Accepted: 22 March 2021 / Published online: 17 April 2021

(c) The Author(s) 2021

To the Editor,

In patients with asthma, low forced expiratory volume in 1 second $\left(\mathrm{FEV}_{1}\right)$ values represent objectively measurable, strong independent predictors of a future risk of exacerbations [1-3]. Thus, an important treatment outcome in patients with asthma is to achieve normal or near-normal lung function.

The Global Initiative for Asthma 2020 recommends treatment with medium-dose inhaled corticosteroids (ICS) with long-acting $\beta_{2}$-agonists (LABA) as the preferred controller for patients with asthma at step 4 and high-dose ICS with LABA for step 5 [1]. However, some patients remain inadequately controlled despite treatment with LABA/ ICS medium- or high-dose. Add-on treatment with a longacting muscarinic antagonist (LAMA) can provide further benefits in these patients [4-6]. Indacaterol acetate/glycopyrronium bromide/mometasone furoate (IND/GLY/MF), delivered via Breezhaler ${ }^{\circledR}$, is a novel once-daily treatment.

Ivan Nikolaev

ivan.nikolaev@novartis.com

1 Division of Respiratory Medicine, Department of Medicine, University of Toronto, Toronto, Canada

2 Asthma \& Airway Centre, University Health Network, Toronto, Canada

3 Pulmonary Research Institute at LungenClinic Grosshansdorf, Airway Research Center North (ARCN), German Center for Lung Research (DZL), Grosshansdorf, Germany

4 Medicines Evaluation Unit, University of Manchester, Manchester University NHS Foundation Trust, Manchester, UK

5 Fraunhofer Institute of Toxicology and Experimental Medicine and Respiratory Medicine of Hannover Medical
The LABA/LAMA/ICS combination is approved for the maintenance treatment of asthma [7].

In this post-hoc analysis of data from two multicentre trials, we compared the effect of treatment interventions on lung function in a responder analysis. Normal lung function was defined as $\mathrm{FEV}_{1}$ area under the curve from 0 to $24 \mathrm{~h}\left[\mathrm{AUC}_{(0-24 \mathrm{~h})}\right] \geq 90 \%$ of predicted normal after treatment and near-normal lung function was defined as $\mathrm{FEV}_{1}$ $\left[\mathrm{AUC}_{(0-24 \mathrm{~h})}\right] \geq 80 \%$ of predicted normal after treatment. We report results from a responder analysis evaluating the percentage of patients achieving normal or near-normal lung function with IND/GLY/MF compared with salmeterol xinafoate/fluticasone propionate (SAL/FLU) or placebo from two phase II studies (CQVM149B2208 [7], NCT03063086; CQVM149B2209 [8], NCT03108027).

CQVM149B2208 (hereafter referred to as B2208) was a randomised, double-blind, double-dummy, active-controlled, three-period (21 days each), six-sequence crossover study in patients with moderate-to-severe asthma. Eligible patients were randomised to one of the six possible

School, Member of the German Center for Lung Research (DZL), Hannover, Germany

6 Department of Respiratory Medicine and Allergology, Institute for Clinical Science, Skane University Hospital, Lund University, Lund, Sweden

7 Department of Microbiology Immunology and Transplantation, KU Leuven, Catholic University of Leuven, Leuven, Belgium

8 Department of Clinical Pharmacy and Pharmacology, UMCG and QPS Netherlands, Groningen, The Netherlands

9 Novartis Pharma AG, 4002 Basel, Switzerland

10 Novartis Institutes for BioMedical Research, Translational Medicine, Basel, Switzerland 
treatment sequences, to receive IND/GLY/MF high-dose ICS $(150 / 50 / 160 \mu \mathrm{g})$ or IND/GLY/MF medium-dose ICS $(150 / 50 / 80 \mu \mathrm{g})$ once daily delivered via Breezhaler ${ }^{\circledR}$ or SAL/FLU high-dose ICS $(50 / 500 \mu \mathrm{g})$ twice daily delivered via Accuhaler ${ }^{\circledR}$, for 21 days in each of the three treatment periods.

CQVM149B2209 (hereafter referred to as B2209) was a randomised, double-blind, placebo-controlled, three-period (14 days each), six-sequence crossover study in patients with mild-to-moderate asthma. Eligible patients were randomised to one of the six possible treatment sequences, to receive IND/GLY/MF medium-dose once daily in the evening or morning, delivered via Breezhaler ${ }^{\circledR}$ or placebo via a matching inhaler, for 14 days in each of the three treatment periods.

Male and female patients (aged $\geq 18$ years) with a prebronchodilator $\mathrm{FEV}_{1}$ of $<80 \%$ of the predicted value were included in the B2208 study, and those with a pre-bronchodilator $\mathrm{FEV}_{1}$ of $\geq 60 \%$ and $<100 \%$ of the predicted value were included in the B2209 study. Both studies included patients with an $\mathrm{FEV}_{1}$ increase of $\geq 12 \%$ and $>200 \mathrm{~mL}$ after administration of $400 \mu \mathrm{g}$ of salbutamol or $360 \mu \mathrm{g}$ of albuterol. Patients with an asthma exacerbation requiring systemic steroids, hospitalisation or an emergency room visit within 6 weeks prior to the study were excluded. Detailed information on the study designs and patient criteria is described in the respective publications [7, 8]. Patients included in the B2209 study had less severe lung function impairment with a pre-bronchodilator $\mathrm{FEV}_{1}$ between 60 and $100 \%$ of the predicted value at screening. As the "nearnormal" cut-off of $\geq 80 \%$ falls in the middle of the range required at inclusion, some patients were already above it, and therefore the criterion to assess improvement was raised to $\geq 90 \%$ ("normal"). However, the B2208 study included patients with $\mathrm{FEV}_{1}<80 \%$ at screening, therefore achieving "near-normal" lung function could be assessed in B2208.

In the B2208 study, both IND/GLY/MF high-dose and medium-dose demonstrated greater improvements in peak $\mathrm{FEV}_{1}$ vs SAL/FLU after 21 days of treatment. IND/GLY/ $\mathrm{MF}$ at both ICS doses provided greater improvements in trough $\mathrm{FEV}_{1}$ and forced vital capacity $\mathrm{AUC}_{0-24 \mathrm{~h}}$ compared with SAL/FLU. Mean forced expiratory flow at 25-75\% also improved with IND/GLY/MF high-dose and mediumdose $(1.71 \mathrm{~L} / \mathrm{s}$ and $1.70 \mathrm{~L} / \mathrm{s}$, respectively) compared with SAL/FLU (1.54 L/s).

Similarly, in the B2209 study, medium-dose IND/GLY/ MF showed greater improvement in $\mathrm{FEV}_{1}\left(\mathrm{AUC}_{0-24 \mathrm{~h}}\right)$ for both morning and evening administrations vs placebo after 14 days of treatment [8]. Greater improvements in trough $\mathrm{FEV}_{1}$ were also seen with medium-dose IND/GLY/MF, regardless of the time of administration (morning or evening), and provided substantial improvements in forced vital capacity $\left(\mathrm{AUC}_{0-24 \mathrm{~h}}\right)$ compared with placebo.
In the B2208 study, $44.6 \%$ and $47.3 \%$ of patients taking IND/GLY/MF high- and medium-dose, respectively, achieved near-normal lung function compared with $33.7 \%$ of patients taking SAL/FLU (Fig. 1a). In the B2209 study, $47.0 \%$ of patients taking IND/GLY/MF mediumdose achieved normal lung function vs $6.7 \%$ patients

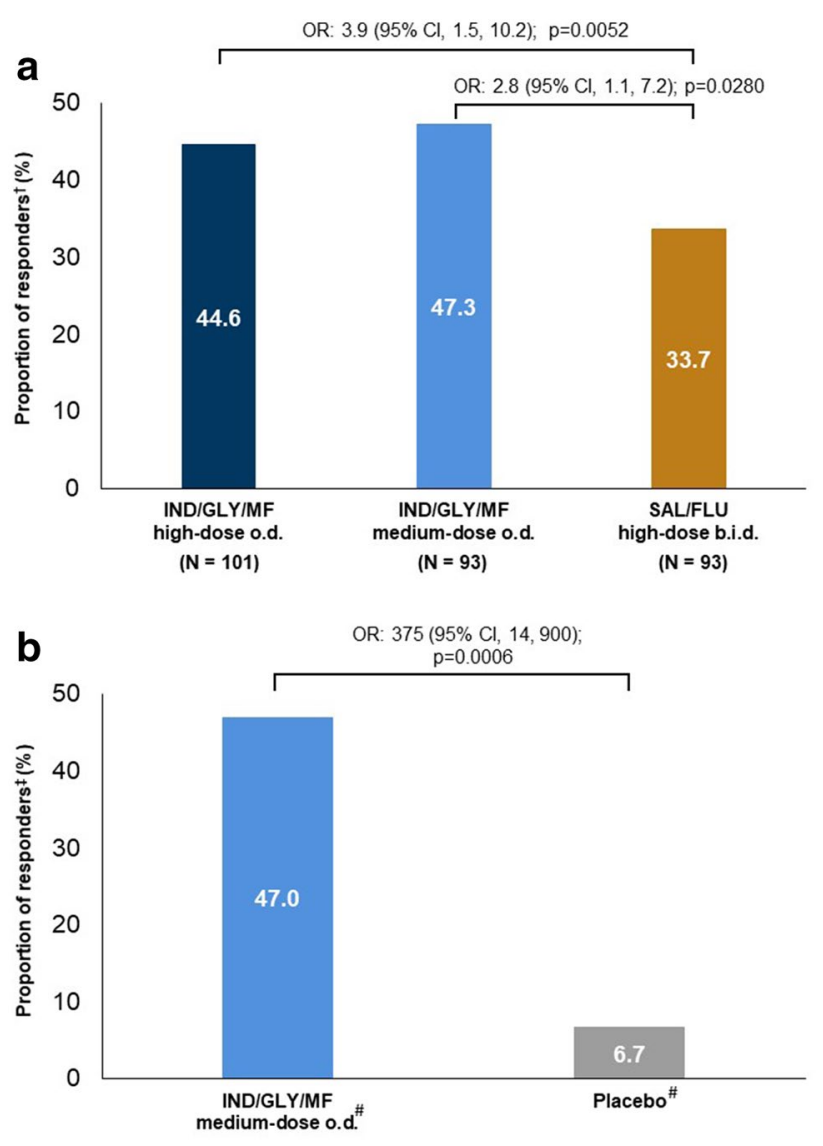

Fig. 1 Lung function normalisation with indacaterol acetate/glycopyrronium bromide/mometasone furoate (IND/GLY/MF) in patients with asthma. a Near-normal lung function (forced expiratory volume in 1 second area under the curve from 0 to 24 hours [FEV $\left.\mathrm{AUC}_{(0-24 \mathrm{~h})}\right] \geq 80 \%$ predicted) with IND/GLY/MF high-dose and medium-dose vs salmeterol/fluticasone (SAL/FLU) high-dose and $\mathbf{b}$ normal lung function $\left(\mathrm{FEV}_{1} \mathrm{AUC}_{(0-24 \mathrm{~h})} \geq 90 \%\right.$ predicted) with IND/ GLY/MF medium-dose vs placebo. A.M. morning, b.i.d. twice daily, $C I$ confidence interval, o.d. once daily, $O R$ odds ratio, P.M. evening. ${ }^{\#} N=33$ and $N=31$ patients were included in the o.d. IND/GLY/MF medium-dose A.M. and P.M. groups, respectively; $N=30$ patients were included in the placebo group. Responders' data presented here represent the mean of percentage responders from IND/GLY/ MF medium-dose A.M. and P.M. groups. 'Response for a treatment period is defined as achieving $\mathrm{AUC}_{(0-24 \mathrm{~h})}$ of predicted $\mathrm{FEV}_{1} \geq 80 \%$ after the end of the treatment period in B2208. ${ }^{+}$Response for a treatment period is defined as achieving $\mathrm{AUC}_{(0-24 \mathrm{~h})}$ of predicted $\mathrm{FEV}_{1} \geq$ 90\% after the end of the treatment period in B2209. Responder (yes/ no) is analysed using a logistic regression with treatment, sequence, period as factors having fixed effects, baseline pre-bronchodilator $\%$ predicted $\mathrm{FEV}_{1}$ as a covariate having a fixed effect and subject having a random effect 
receiving placebo (Fig. 1b). This was irrespective of the time of dose (morning or evening), as the percentage of patients who achieved normal lung function with mediumdose IND/GLY/MF at morning and evening administrations was $45.2 \%$ and $48.5 \%$, respectively, compared with $6.7 \%$ receiving placebo.

Studies in asthma suggest that improvements in the peak expiratory flow (PEF) of $15-20 \mathrm{~L} / \mathrm{min}$ are clinically relevant and perceptible by patients $[9,10]$. The lung function normalisation outcomes, which were achieved with IND/GLY/MF vs SAL/FLU and placebo, were complemented by a greater proportion of patients achieving clinically meaningful improvement in PEF with IND/ GLY/MF vs these comparators. In the B2208 study, 63.6\% and $63.2 \%$ of patients taking IND/GLY/MF high-dose and medium-dose, respectively, had an improvement of $\geq 20 \mathrm{~L} / \mathrm{min}$ in PEF vs $31.0 \%$ of patients taking SAL/FLU. In the B2209 study, a greater percentage of patients treated with IND/GLY/MF medium-dose $(91.0 \%$ and $84.4 \%$ for morning and evening doses, respectively) had an improvement of $\geq 20 \mathrm{~L} / \mathrm{min}$ in PEF vs placebo (11.4\%).

Furthermore, lung function normalisation with IND/ GLY/MF was complemented by a greater proportion of patients with no rescue medication use with IND/GLY/ MF vs SAL/FLU and placebo. In the B2208 study, a higher percentage of patients treated with IND/GLY/MF highdose (58\%) did not require rescue medication compared with patients treated with SAL/FLU (45\%) [odds ratio: 2.4; 95\% confidence interval [CI] 1.7-5.0; $p=0.018]$; a similar trend was also observed with IND/GLY/MF medium-dose (52\%; OR: $1.7 ; 95 \%$ CI $0.8-3.4 ; p=0.1530$ ) vs SAL/FLU [7]. A substantial proportion of patients did not require rescue medication with either morning (67.6\%; OR: 11.07; 95\% CI 3.08-39.81; $p=0.0015$ ) or evening (70.6\%; OR: 11.96; 95\% CI 3.45-41.39; $p=0.0016)$ administration of IND/GLY/MF medium-dose compared with placebo in the B2209 study. Regardless of the timing of administration, for a given patient, the odds of being "rescue medication free" were higher when treated with IND/GLY/MF medium-dose compared with placebo.

Lung function normalisation can be assessed by improvements in $\mathrm{FEV}_{1}\left(\mathrm{AUC}_{0-24 \mathrm{~h}}\right)$, supported by improvements observed in other lung function parameters. Another important goal of asthma treatment is to eliminate or reduce the need for rescue medication [2].

Overall, results from B2208 and B2209 studies demonstrated greater improvements in lung function with IND/ GLY/MF compared with SAL/FLU and placebo, respectively. These improvements in lung function translated into more patients achieving normal or near-normal lung function with IND/GLY/MF compared with SAL/FLU and placebo. In addition, patients were more likely to be rescue medication free with IND/GLY/MF compared with highdose SAL/FLU or placebo.

A responder analysis based on the ability to achieve normal lung function helps to translate clinical trial results into clinically useful terms. However, at least one limitation of this approach must be noted. Neither B2208 nor B2209 studies allowed us to define the best achievable lung function, an alternative and acceptable endpoint in the clinical setting if remodelling prevents the achievement of populationpredicted normal values.

In summary, results from this responder analysis, based on the lung function normalisation and reduction in rescue medication use, provide further evidence on benefits of IND/ GLY/MF in patients with asthma and support the use of this LABA/LAMA/ICS combination in patients who are inadequately controlled on LABA/ICS.

Acknowledgements The authors thank Venkatesh Taadla and Vatsal Vithlani (Novartis Healthcare Pvt. Ltd.) for providing scientific writing support for this article, which was funded by Novartis Pharma AG, Basel, Switzerland in accordance with Good Publication Practice (GPP3) guidelines (http://www.ismpp.org/gpp3). Dave Singh is supported by the National Institute for Health Research Manchester Biomedical Research Centre.

\section{Declarations}

Funding The studies were funded by Novartis Institutes for BioMedical Research. The funder had a role in the study design, data collection, data analysis and interpretation, and writing of the manuscript.

Conflicts of interest Kenneth R. Chapman reports grants and personal fees from AstraZeneca, Boehringer Ingelheim, Grifols, GlaxoSmithKline, Sanofi, Regeneron, Novartis and Takeda. Kenneth R. Chapman also received personal fees from CSL Behring, Inhibrx and Kamada, and grants from Vertex, outside the submitted work. Henrik Watz reports grants, personal fees and non-financial support from Novartis, during the conduct of the study; grants, personal fees and non-financial support from AZ, BerlinChemie/Menarini, GlaxoSmithKline, Chiesi, Bayer and Takeda, outside the submitted work. Dave Singh reports personal fees from AstraZeneca, Boehringer Ingelheim, Chiesi, Cipla, Genentech, GlaxoSmithKline, Glenmark, Gossamerbio, Menarini, Mundipharma, Novartis, Peptinnovate, Pfizer, Pulmatrix, Theravance and Verona, outside the submitted work. Jens M. Hohlfeld reports grants from Novartis during the conduct of the study. Jens M. Hohlfeld also reports personal fees from Boehringer Ingelheim, Merck \& Co, Inc., Novartis and HAL; and grants from AstraZeneca AB, Novartis, Janssen Pharmaceutica NV, ALK, Boehringer Ingelheim, LETI, GlaxoSmithKline, Sanofi-Aventis, Astellas Pharma and Allergopharma, outside the submitted work. In the past 3 years, Zuzana Diamanat acted as the Research Director at QPS-NL, an institution that received research support from several bio-pharmaceutical companies, especially within respiratory field: Patara Pharma (now Respivant), Novartis, Foresee Pharmaceuticals. Furthermore, Zuzana Diamanat received honoraria or speaker fees for serving on advisory boards or acting as a consultant from ALK, AstraZeneca, Boehringer Ingelheim, GlaxoSmithKline, HAL Allergy, Merck Sharp \& Dohme and Sanofi-Genzyme-Regeneron. In addition, Zuzana Diamanat also acts as an advisor for BMR BV, which holds patents for several respiratory indications. Ieuan Jones and 
Ivan Nikolaev are employees of Novartis. Hanns-Christian Tillmann is an employee of Novartis Institutes for Biomedical Research and owns Novartis shares.

Ethics approval The study was conducted in accordance with the Declaration of Helsinki and was approved by the independent ethics committees of participating sites in Europe and China.

Consent to participate Written informed consent was obtained from each patient before conducting any study-specific procedures.

\section{Consent for publication Not applicable.}

Availability of data and material Novartis is committed to sharing with qualified external researchers access to patient-level data and supporting clinical documents from eligible studies. These requests are reviewed and approved by an independent review panel on the basis of scientific merit. All data provided are anonymized to respect the privacy of patients who have participated in the study in line with applicable laws and regulations. This study data availability is according to the criteria and process described on www.clinicalstudydatareq uest.com.

Code availability Not applicable.

Authors' contributions KRC, IJ, HCT and IN conceptualised this responder analysis. HW, DS, JHM, ZD and HCT designed the studies. HW, DS, JHM and ZD were responsible for data acquisition. IJ was involved in the extraction of the data and statistical analyses. All the authors were involved in the interpretation of the results and the writing and critical revision of the manuscript with the support of professional medical writers mentioned in the Acknowledgements section. All authors provided final approval on the version to be submitted.

Open Access This article is licensed under a Creative Commons Attribution-NonCommercial 4.0 International License, which permits any non-commercial use, sharing, adaptation, distribution and reproduction in any medium or format, as long as you give appropriate credit to the original author(s) and the source, provide a link to the Creative Commons licence, and indicate if changes were made. The images or other third party material in this article are included in the article's Creative Commons licence, unless indicated otherwise in a credit line to the material. If material is not included in the article's Creative Commons licence and your intended use is not permitted by statutory regulation or exceeds the permitted use, you will need to obtain permission directly from the copyright holder. To view a copy of this licence, visit http://creativecommons.org/licenses/by-nc/4.0/.

\section{References}

1. Global Initiative for Asthma. Global strategy for asthma management and prevention. 2020. http://ginasthma.org/gina-reports/. Accessed 30 Sep 2020.

2. Khan A, Kamat S, Eckert L, et al. Impact of baseline lung function on future exacerbations in patients with moderate-to-severe asthma. Ann Allergy Asthma Immunol. 2018;121:S22-62 (poster session: P220).

3. Kitch BT, Paltiel AD, Kuntz KM, et al. A single measure of $\mathrm{FEV}_{1}$ is associated with risk of asthma attacks in long-term follow-up. Chest. 2004;126(6):1875-82.

4. Demoly P, Paggiaro P, Plaza V, et al. Prevalence of asthma control among adults in France, Germany, Italy, Spain and the UK. Eur Respir Rev. 2009;18:105-12.

5. Partridge MR, Dal Negro RW, Olivieri D. Understanding patients with asthma and COPD: insights from a European study. Prim Care Respir J. 2011;20:315-23.

6. Kew KM, Dahri K. Long-acting muscarinic antagonists (LAMA) added to combination long-acting beta2-agonists and inhaled corticosteroids (LABA/ICS) versus LABA/ICS for adults with asthma. Cochrane Database Syst Rev. 2016;1:CD011721.

7. Watz H, Hohlfeld JM, Singh D, et al. Indacaterol/glycopyrronium/ mometasone furoate compared with salmeterol/fluticasone propionate in patients with asthma: a randomized controlled cross-over study. Respir Res. 2020;21(1):87.

8. Beier J, Watz H, Scholz V, et al. Lung function improvements following inhaled indacaterol/glycopyrronium/mometasone furoate are independent of dosing time in asthma patients: a randomised trial. ERJ Open Res. 2020. https://doi.org/10.1183/23120541. 00425-2020.

9. Santanello NC, Zhang J, Seidenberg B, et al. What are minimal important changes for asthma measures in a clinical trial? Eur Respir J. 1999;14(1):23-7.

10. Reddel HK, Taylor DR, Bateman ED, et al. An official American Thoracic Society/European Respiratory Society statement: asthma control and exacerbations: standardizing endpoints for clinical asthma trials and clinical practice. Am J Respir Crit Care Med. 2009;180(1):59-99. 